\title{
Effect of degradation of PLGA and PLGA/ $\beta$-TCP scaffolds on the growth of osteoblasts
}

\author{
YANG YanFang ${ }^{1}$, TANG GongWen ${ }^{1}$, ZHAO YunHui ${ }^{1}$, ZHANG Yang $^{2}$, LI XiuLan ${ }^{2}$ \& \\ YUAN XiaoYan ${ }^{1 *}$ \\ ${ }^{1}$ School of Materials Science and Engineering, Tianjin Key Laboratory of Composite and Functional Materials, Tianjin University, Tianjin \\ 300072, China; \\ ${ }^{2}$ Institute of Orthopedics, Tianjin Hospital, Tianjin 300211, China
}

Received April 8, 2010; accepted August 4, 2010; published online November 1, 2010

\begin{abstract}
Osteoblasts were cultured on porous scaffolds of poly(L-lactide-co-glycolide) (PLGA) and PLGA/ $\beta$-tricalcium phosphate ( $\beta$-TCP) to evaluate their cytocompatibility. The proliferation of the cells on both scaffolds was examined before and after in vitro degradation for 4, 8 and 12 weeks under static (shaking water bath) and dynamic (cyclic loading) conditions. Results indicate that porous PLGA and PLGA/ $\beta$-TCP scaffolds have good biocompatibility and can be used as effective templates for guiding the growth of osteoblasts. The degradation of the scaffolds affects the proliferation of osteoblasts and the cell viability decreased with the degradation time.
\end{abstract}

PLGA, $\beta$-TCP, scaffold, degradation, osteoblast

Citation: Yang Y F, Tang G W, Zhao Y H, et al. Effect of degradation of PLGA and PLGA/ $\beta$-TCP scaffolds on the growth of osteoblasts. Chinese Sci Bull, 2011, 56: 982-986, doi: 10.1007/s11434-010-4132-1

Because of the limitations of autografts and allografts, many efforts have been made over the past two decades to prepare ideal bone repair biomaterials from the viewpoint of tissue engineering [1]. One of the most important objectives of bone tissue engineering is to design and develop adequate biodegradable scaffolds for cells to adhere, proliferate and differentiate, in addition to guiding the formation of bone tissue [2]. Polylactide, polyglycolide and their copolymer poly(L-lactide-co-glycolide) (PLGA) are widely used in bone tissue engineering because of their excellent biodegradability [2]. $\beta$-tricalcium phosphate $(\beta$-TCP) is also widely applied in the regeneration of the bone tissue because of its good biocompatibility and osteoinductive activity [3].

Shen et al. [2] reported that osteoblast-like cells could grow and differentiate well on PLGA/hydroxyapatite scaffolds containing bone morphogenetic protein-2. In vitro cell

*Corresponding author (email: yuanxy@tju.edu.cn) proliferation studies of human fetal osteoblasts seeded on poly( $\varepsilon$-caprolactone $) / \beta$-TCP scaffolds showed the great potential of these scaffolds that can mimic the complex elegance of native tissue [3]. Furthermore, Kuo et al. [4] reported that bone marrow stromal cells grew well on PLGA/chitosan scaffolds, indicating that biodegradable porous scaffolds can supply a three-dimensional template for cell growth. The degradability of the scaffolds may also effect the growth of the cells; however, there are too few reports on the cultivation of cells on the scaffolds over different degradation periods.

In this work, osteoblasts were cultured on porous PLGA and PLGA/ $\beta$-TCP scaffolds to evaluate the cytocompatibility of the scaffolds. The proliferation of the cells on both scaffolds, after degradation for 4, 8 and 12 weeks, was examined under static (shaking water bath) and dynamic (cyclic loading) conditions. This study specifically investigates the biocompatibility of the scaffolds over different degradation times. 


\section{Materials and methods}

(i) Materials. PLGA (LA/GA $=70 / 30, \quad \bar{M}_{n}=1.57 \times 10^{5}$ ) was donated by the Changchun Institute of Applied Chemistry, Chinese Academy of Sciences, China. $\beta$-TCP $\left(d_{50}=3.22 \mu \mathrm{m}\right)$ was supplied by the National Engineering Research Centre for Biomaterials, Sichuan University, China. Commercially-obtained sucrose particles, sieved into $200-400 \mu \mathrm{m}$, were used as a porogen. Osteoblasts were obtained from the skull of neonatal Sprague-Dawley rats and passaged four times prior to use. Fetal bovine serum and Dulbecco's-modified eagles medium (DMEM) was purchased from Gibco. 3-(4,5-dimethythiazol-2-yl)-2,5-dipheyl tetrazolium bromide (MTT) was supplied by Sigma. Dimethylsulfoxide (DMSO) was supplied by Tianjin Fangde Technology Company, China. D-Hank's buffer solution was provided by Tianjin Unit Star Biotechnology Co. Ltd., China. 2\% paraformaldehyde- $2 \%$ glutaraldehyde (2\% PFA$2 \%$ GA) was purchased from Tianjin Chemical Agent Institute, China. Alkaline phosphatase (ALP) kit was purchased from Randox. All the chemicals used were of analytical quality grade throughout the study.

(ii) Preparation of porous scaffolds. Porous PLGA and PLGA/ $\beta$-TCP scaffolds were fabricated using a technique combining thermally-induced phase separation and porogen leaching [5]. Briefly, PLGA was dissolved in chloroform to form a homogeneous $0.15 \mathrm{~g} / \mathrm{mL}$ solution and blended with sucrose particles ( $85 \mathrm{vol} \%$ ). The mixture of PLGA solution and porogen was poured into a custom-made cylindrical Teflon mold and frozen at $-18^{\circ} \mathrm{C}$ to induce phase separation for $12 \mathrm{~h}$. Subsequently, the solvent (chloroform) was extracted using ethanol, and the porogen was leached from the solution using distilled water. After drying, porous cylindrical PLGA scaffolds were obtained. For the preparation of PLGA/ $\beta$-TCP scaffolds, a certain amount of $\beta$-TCP (40 wt $\%$ of PLGA) was added to the clear PLGA solution and dispersed using ultrasonic stirring prior to the addition of the porogen. The preparation process was identical with the PLGA scaffold. The morphology of all samples was observed by scanning electron microscopy (SEM) (Philips XL30), after gold-coating. PLGA and PLGA//3-TCP scaffolds (6 $\mathrm{mm}$ diameter, $3 \mathrm{~mm}$ height) were examined before and after degradation under static and dynamic conditions $[6,7]$. The scaffolds were sterilized by irradiation using $\mathrm{Co}^{60}$ at an intensity of $15 \mathrm{kGy}$ prior to cell culture.

(iii) MTT assay. PLGA and PLGA/ $\beta$-TCP scaffolds were placed into 96-well culture plates and seeded with a cell suspension $(100 \mu \mathrm{L})$, containing cells at a density of $5 \times 10^{4}$ cells $/ \mathrm{mL}$, followed by culturing for $14 \mathrm{~d}$. The culture medium was changed every other day. At pre-determined intervals $(2,4,7$ and $14 \mathrm{~d})$, the culture medium was removed for measurement of MTT values in an automatic enzyme-linked immunosorbent assay (Dynex-MEX) at 570 $\mathrm{nm}$. The mean and standard deviation were obtained from six parallel samples. The scaffolds were numbered as PLGA (PLGA scaffolds before degradation), PLGA-S4 (PLGA scaffolds after degradation for 4 weeks under static conditions), PLGA-D4 (PLGA scaffolds after degradation for 4 weeks under dynamic conditions), PLGA/ $\beta$-TCP (PLGA/ $\beta$ TCP scaffolds before degradation), PLGA/ $\beta$-TCP-S4 (PLGA/ $\beta$-TCP scaffolds after degradation for 4 weeks under static conditions), PLGA/ $\beta$-TCP-D4 (PLGA/ $\beta$-TCP scaffolds after degradation for 4 weeks under dynamic conditions) and so on.

(iv) ALP activity. PLGA and PLGA/ $\beta$-TCP scaffolds were placed into a 96-well cell culture plate, and the cell suspension $(100 \mu \mathrm{L})$ with a cell density of $2 \times 10^{5} \mathrm{cells} / \mathrm{mL}$ was seeded evenly into the scaffolds for $14 \mathrm{~d}$. The culture medium was changed two times every week. At day 7 and 14 of the incubation, the cells were digested by trypsin/ EDTA after discarding the supernatant, followed by three cycles of freezing and thawing $\left(-80^{\circ} \mathrm{C}-\right.$ room temperature $)$. The ALP activity of the supernatant was obtained by centrifugation and determined colorimetrically, using an ALP kit in Automatic Biochemistry Analyzer (Toshiba, Japan). The mean and the standard deviation were obtained from three parallel samples.

(v) Cell morphology. Cell suspension $(50 \mu \mathrm{L}$ in a density of $2 \times 10^{6}$ cells $/ \mathrm{mL}$ ) was seeded onto the PLGA and PLGA/ $\beta$-TCP scaffolds prior to degradation. After culturing in an incubator $\left(5 \% \mathrm{CO}_{2}, 37^{\circ} \mathrm{C}\right)$ for $4 \mathrm{~h}$, another $100 \mu \mathrm{L}$ cell culture medium was added to each well of the 96-well plate. After 7 and $14 \mathrm{~d}$ of culture, the samples were fixed by $2 \%$ PFA-2\% GA, after washing with D-Hank's buffer. The samples were then dehydrated through a series of graded alcohols, freeze-dried, and sputter-coated with gold. Finally, the morphology of the cells on the scaffolds was observed by SEM.

\section{Results and discussion}

\subsection{PLGA and PLGA/ $\beta$-TCP scaffolds before and after degradation}

PLGA and PLGA/ $\beta$-TCP scaffolds were degraded under static and dynamic conditions for 4,8 and 12 weeks. Previous studies showed that degradation of PLGA becomes increasingly remarkable with the degradation time, and a further increase in degradation happens under dynamic conditions [6,7]. Figure 1 shows the SEM micrographs of PLGA and PLGA/ $\beta$-TCP scaffolds before and after degradation for 12 weeks, under static and dynamic conditions. The results indicate that the pore size of both PLGA (Figure 1(a)) and PLGA/ $\beta$-TCP scaffolds (Figure 1(b)) was mainly distributed in the range of $100-350 \mu \mathrm{m}$. The porosity of the two scaffolds was approximately $90 \%$, as determined by modified ethanol replacement method [5]. The SEM micrograph 

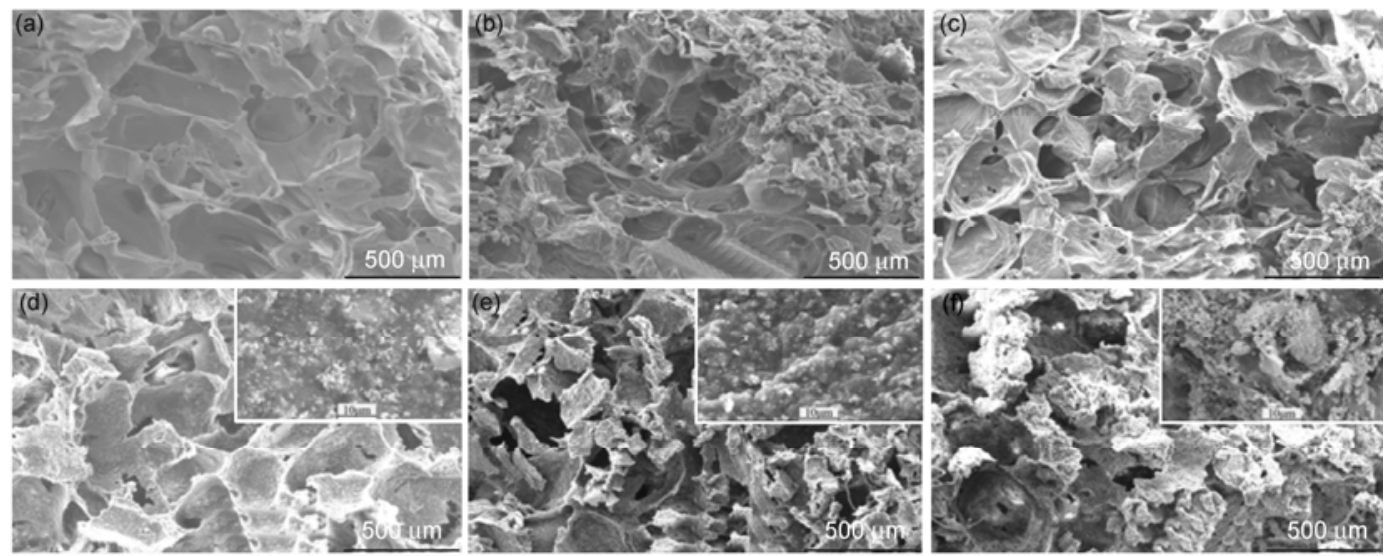

Figure 1 SEM micrographs of PLGA $(a, b, c)$ and PLGA/ $\beta-T C P(d, e, f)$ scaffolds, before $(a, d)$ and after degradation for 12 weeks $(b, c, e, f)$ under static $(b, e)$ and dynamic (c,f) conditions.

of the PLGA/ $\beta$-TCP scaffold (Figure 1(d)) suggests that there is an even distribution of $\beta$-TCP on the pore wall of the scaffold. The mass percentage of $\beta$-TCP in PLGA/ $\beta$ TCP scaffolds was determined by weighing the $\beta$-TCP amount obtained from the PLGA/ $\beta$-TCP scaffold/chloroform solution after high speed centrifugation and vacuumdried, showing that the mass percentage of $\beta$-TCP in the scaffold was about $42 \%$ [7]. The mass, relative molecular mass, size and mechanical properties of both scaffolds decreased with increasing degradation time, which coincides with the results of $\mathrm{Wu}$ et al. [8,9]. The mass percentage of $\beta$-TCP in the scaffolds showed an increased tendency with the degradation time [7].

\subsection{Comparative study of the cell viability on PLGA and PLGA/ $\beta$-TCP scaffolds}

(i) MTT assay. MTT assay was used to detect the cell viability of the osteoblasts on the scaffolds, with the results shown in Figure 2. It can be seen that the cells could proliferate well on both the PLGA and PLGA/ $\beta$-TCP scaffolds. The cells on the PLGA/ $\beta$-TCP scaffolds had a lower viability than on the PLGA scaffolds. It may be caused by the introduction of $\beta$-TCP particles, which possibly changed the surface properties of the porous scaffolds. The physicochemical properties of biomaterials, such as topography, hydrophobicity, charge and free energy, can affect cell adhesion and proliferation when seeded on scaffolds [10]. Furthermore, the introduction of $\beta$-TCP may cause a decrease in the total surface area of the scaffolds, leading to a decrease in the available space for cell proliferation.

(ii) ALP activity. Figure 3 presents the ALP activity of osteoblasts on PLGA scaffolds and PLGA/ $\beta$-TCP scaffolds after culturing for 7 and $14 \mathrm{~d}$. The results indicate that the ALP activity of osteoblasts on both scaffolds increased with prolonged culture time, with similar results for both types of scaffolds. The results also demonstrate that the introduction

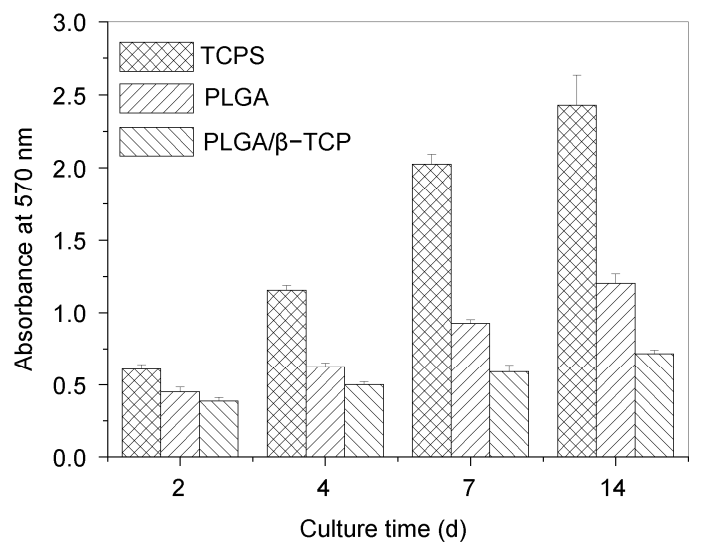

Figure 2 Proliferation of osteoblasts on PLGA and PLGA/ $\beta$-TCP scaffolds.

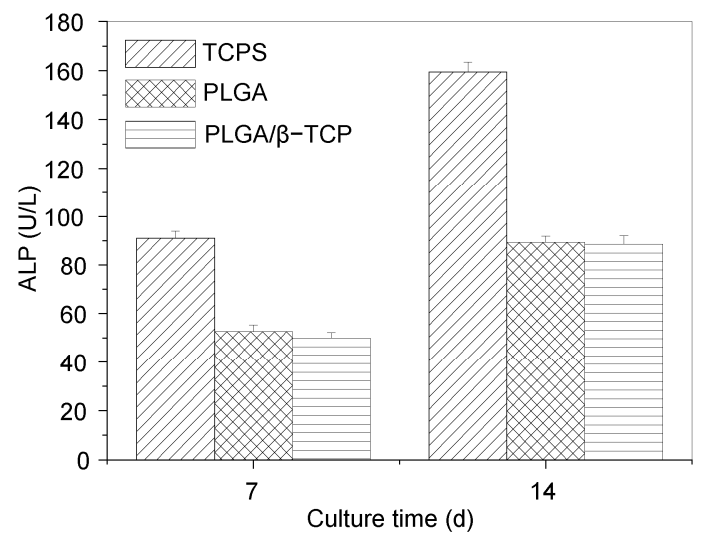

Figure 3 ALP activity of osteoblasts on PLGA and PLGA/ $\beta$-TCP scaffolds.

of $\beta$-TCP into the porous scaffolds does not significantly promote cell differentiation.

There was no obvious difference in the ALP activity of osteoblasts on both scaffolds, which differs from a report that claimed the introduction of biological mineralized apatite 
had positive effects on the differentiation of osteoblasts [11]. This may be explained by the differences in bone-like apatite used in various studies, as different preparation methods could cause variations in the physical properties of apatite, such as crystallinity, crystal size, surface topography, elemental composition/ratio and the dissolution rate of apatite. Each of these properties could substantially influence the attachment, proliferation and differentiation of osteoblasts in vitro [12]. Kim et al. [13] also reported that the ALP activity of osteoblast-like cells on the hydroxyapatite coating of zirconia porous scaffolds was increased in comparison with those on pure $\beta$-TCP, with higher dissolution rates. In this study, the introduction of $\beta$-TCP particles did not promote cell differentiation significantly, which may be related to the relatively large particle size of $\beta$-TCP and the short cell incubation time. The ALP activity of osteoblasts on PLGA/ $\beta$-TCP scaffolds may increase with culture time and $\beta$-TCP could promote osteogenic differentiation with prolonged cell incubation.

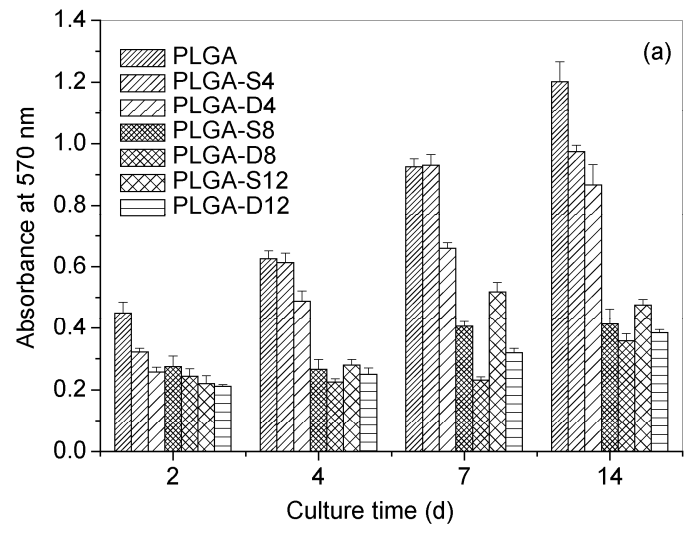

\subsection{Effects of degradation of PLGA and PLGA/ $\beta$-TCP scaffolds on cell viability}

Figure 4 shows the proliferation of osteoblasts on PLGA and PLGA $/ \beta$-TCP scaffolds, before and after degradation for 4, 8 and 12 weeks, under static and dynamic conditions. The results indicate that the cells could proliferate on both scaffolds, with the highest MTT values on both scaffolds prior to degradation. The MTT values of cells on the scaffolds decreased with increased degradation of the scaffolds, with the lowest value after degradation for 12 weeks under dynamic conditions. The lower MTT value of the scaffolds after degradation may be caused by the increase of the carboxyl group content on the scaffold surface after degradation. The longer the degradation period, the higher the formation of carboxyl groups on the scaffold surface and the lower the proliferation of cells. In addition, some of the pores had closed after degradation and the aggregation of $\beta$-TCP on the pore wall of the scaffold (Figure 1) may also result in a lower MTT value.

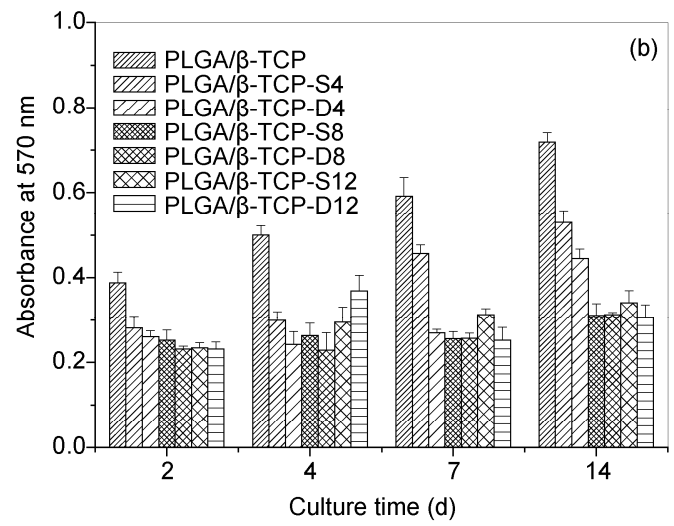

Figure 4 Proliferation of osteoblasts on PLGA (a) and PLGA/ $\beta$-TCP (b) scaffolds after degradation for different time.
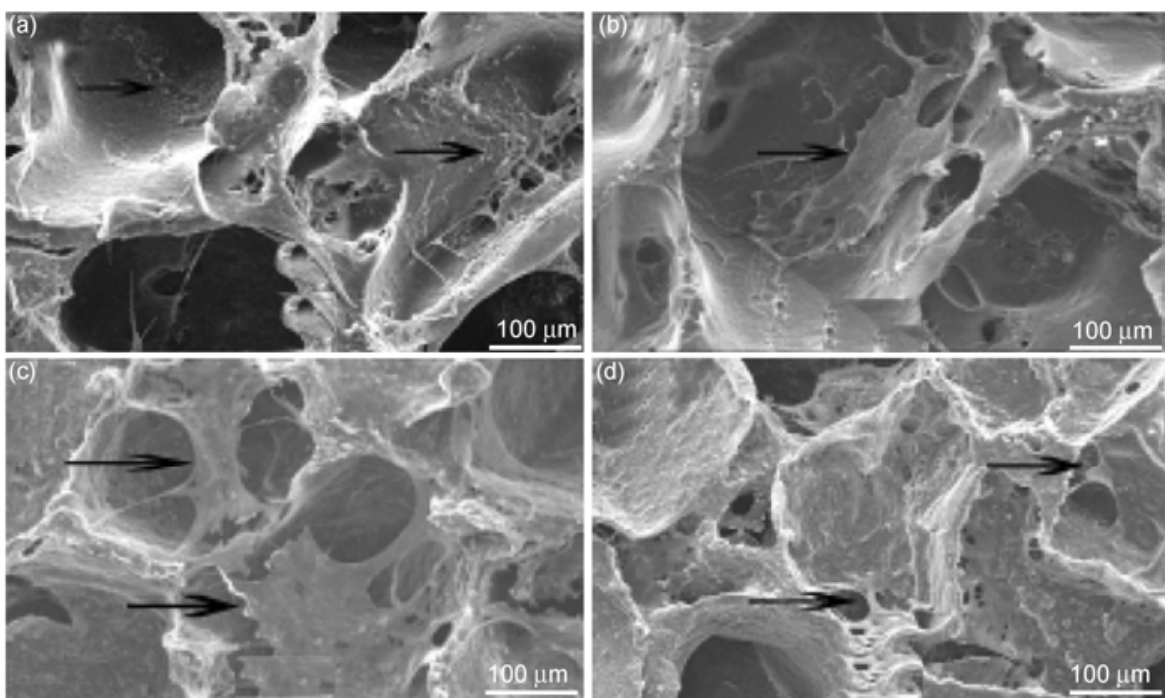

Figure 5 SEM micrographs of PLGA (a,b) and PLGA/ $\beta$-TCP (c,d) scaffolds after cultivation with osteoblasts for 7 (a,c) and $14 \mathrm{~d}(\mathrm{~b}, \mathrm{~d})$. 


\subsection{SEM observation of cell morphology on PLGA and PLGA/ $\beta$-TCP scaffolds}

Figure 5 shows SEM micrographs of PLGA and PLGA/ $\beta$-TCP scaffolds after cultivation for 7 and $14 \mathrm{~d}$. It can be seen that the cells could grow well on both PLGA and PLGA/ $\beta$-TCP scaffolds. Cells had spread into a flat spindle shape with protruded psendepodiums on both scaffolds after incubating for 7 and $14 \mathrm{~d}$, compared with scaffolds before cell culture (arrows in Figure 5). Furthermore, the growing cells had mainly adhered to the pore wall. The cells on the PLGA/ $\beta$-TCP scaffold exhibited a higher degree of spreading after culturing for $7 \mathrm{~d}$ and grew across the pore wall of the scaffolds, as well as adhering to the pore wall.

\section{Conclusions}

Both PLGA and PLGA/ $\beta$-TCP scaffolds show good cell biocompatibility and could serve as effective templates for the culture of osteoblasts. Cells on both scaffolds exhibited good adhesion, proliferation and growth. The degradation of the scaffolds could influence the MTT value of the cells, with a decrease in the value over a longer degradation time. The introduction of $\beta$-TCP particles did not significantly improve cell proliferation or differentiation, even though it was assumed that $\beta$-TCP would promote osteogenic differentiation with prolonged incubation time.

This work was supported by the National Natural Science Foundation of China (10672015 and 30828008).

1 Betz R R. Limitations of autograft and allograft: New synthetic solu- tions. Orthopedics, 2002, 25: 561-570

2 Shen $\mathrm{H}, \mathrm{Hu} X \mathrm{X}$, Yang F, et al. An injectable scaffold: rhBMP2-loaded poly(lactide-co-glycolide)/hydroxyapatite composite microspheres. Acta Biomater, 2010, 6: 455-465

3 Ozkan S, Kalyon D M, Yu X J. Functionally graded beta-TCP/PCL nanocomposite scaffolds: In vitro evaluation with human fetal osteoblast cells for bone tissue engineering. J Biomed Mater Res, 2010, 92A: 1007-1018

4 Kuo Y C, Yeh C F, Yang J T. Differentiation of bone marrow stromal cells in poly(lactide-co-glycolide)/chitosan scaffolds. Biomaterials, 2009, 30: 6604-6613

5 Yang Y F, Zhao J, Zhao Y H, et al. Formation of porous PLGA scaffolds by a combining method of thermally induced phase separation and porogen leaching. J Appl Polym Sci, 2008, 109: 1232-1241

6 Yang Y F, Tang G W, Zhao Y H, et al. Effect of cyclic loading on in vitro degradation of poly(L-lactide-co-glycolide) scaffolds. J Biomater Sci Polym Ed, 2010, 21: 53-66

7 Yang Y F, Tang G W, Zhao Y H, et al. In vitro degradation of porous poly(L-lactide-co-glycolide)/ $\beta$-tricalcium phosphate (PLGA/ $\beta$-TCP) scaffolds under dynamic and static conditions. Polym Degrad Stab, 2008, 93: 1838-1845

$8 \mathrm{Wu}$ L B, Ding J D. In vitro degradation of three-dimensional porous poly(D,L-lactide-co-glycolide) scaffolds for tissue engineering. Biomaterials, 2004, 25: 5821-5830

9 Wu L B, Ding J D. Effects of porosity and pore size on in vitro degradation of three-dimensional porous poly(D,L-lactide-co-glycolide) scaffolds for tissue engineering. J Biomed Mater Res Part A, 2005, 75A: 767-777

10 Rohanizadeh R, Swain M V, Mason R S. Gelatin sponges (Gelfoam $\left.{ }^{\circledR}\right)$ as a scaffold for osteoblasts. J Mater Sci-Mater Med, 2008, 19: $1173-1182$

11 Yu T, Liu Y, Wang Y, Jing X B, et al. Preparation and bioactivity of the composite of PLGA and hydroxyapatite nanocrystals surfacegrafted with L-lactic acid oligomer. Chem J Chinese Univ, 2009, 30: 1439-1444

12 Qu X, Cui W J, Yang F, et al. The effect of oxygen plasma pretreatment and incubation in modified simulated body fluids on the formation of bone-like apatite on poly (lactide-co-glycolide) (70/30). Biomaterials, 2007, 28: 9-18

13 Kim H, Kim H E, Salih V, et al. Dissolution control and cellular responses of calcium phosphate coatings on zirconia porous scaffold. J Biomed Mater Res, 2004, 68A: 522-530

Open Access This article is distributed under the terms of the Creative Commons Attribution License which permits any use, distribution, and reproduction in any medium, provided the original author(s) and source are credited. 